\title{
Unsichtbare Gemeinde — Überlegungen eines Rundfunkpredigers
}

\author{
von Heinrich Suso Braun
}

Wenn es schon für den Pfarrer ein erschreckendes Unterfangen ist, am Sonntag vor seine Gemeinde hinzutreten mit dem Anspruch, „in persona Christi“ zu sprechen und sich so einzumischen in das Verhältnis eines Menschen zu seinem Gott - dann ist dies Erschrecken noch größer, wenn der Sprecher mit dem prophetischen Auftrag zu einer unsichtbaren Gemeinde spricht, die nicht jene Voraussetzungen, jenen Vorschuß an Vertrauen mitbringt wie eine Pfarrgemeinde gegenüber ihrem Pfarrer. Und diese unsichtbare Gemeinde ist von einer unschätzbaren Größe. Ich kenne die Methoden wenig, mit denen man die Zahl der Zuhörer und Zuschauer bei unseren Kommunikationsmitteln feststellen will - die großen Sender rechnen mit Millionen. Mein eigener Raum ist freilich viel geringer: ich spreche seit 27 Jahren jeden Sonntag über Radio Tirol und Vorarlberg und uber den schweizerischen Telefonrundspruch - aber jeder Kontakt mit den Hörern bringt mir die Verantwortung für das sonntägliche Wort zum Bewußtsein.

Wenn ich nun aus dieser Erfahrung Grundsätzliches sagen soll, dann bin ich in Verlegenheit: ich bin kein Theoretiker, wenn mir auch die Erfahrung gewisse Erkenntnisse aufdrängt.

Chesterton stellt einmal die Frage, was man kennen müsse, um dem Hans Latein zu geben. Man antwortet natürlich: Latein. Nein, sagt Chesterton, zuerst muß ich den Hans kennen. Das ist die erste Schwierigkeit des Radiosprechers: der Kirchenprediger kennt seinen "Hans", der Radioprediger aber nicht. Wer sind jene, die sonntags den Rundfunkempfänger auf die Radiopredigt einstellen? Demoskopische Untersuchungen können wohl gewisse Zahlen ermitteln, aber kaum die Besonderheiten der Hörer.

Und doch wird der Radiosprecher - nennen wir ihn Radioprediger, auch wenn dieses Wort fragwürdig geworden ist - zu einem gewissen Kennenlernen kommen, wenn er regelmäßig oder doch mit einer gewissen Regelmäßigkeit vor dem Mikrophon steht. Es bildet sich dann doch so etwas wie eine Gemeinde, die durch den "Mundfunk " noch vergrößert wird. Der Sprecher bekommt dann des öfteren zu hören: Mein Zahnarzt, mein Friseur, dieser und jener hat mich auf ihre Sendung aufmerksam gemacht.

Das ist einer der Gründe, die mir den ständigen Wechsel des Sprechers als nicht zweckmäßig erscheinen lassen - abgesehen davon, daß man zuerst doch Lehrling ist und einige Zeit braucht, bis man den besonderen Anforderungen der Radioverkündigung gerecht wird. Ich glaube auch, daß solche Kenntnis kaum theoretisch zu erwerben ist. Sie kann nur durch die Praxis gewonnen werden.

Ausgesprochene Großgemeinden haben sich im Rundfunk etwa in den dreißiger Jahren schon um Peter Lippert gebildet; wie haben wir damals auf seine Sendungen gewartet. Nach dem Kriege waren es in Bayern Hugo Lang oder in Spanien in noch größerem Ausmaß Venancio Marcos und im italienischen Fernsehen der viel zu früh

P. Dr. Heinrich Suso Braun OFMCap, Innsbruck, ist seit 27 Jahren Rundfunkprediger für verschiedene Sender in Osterreich und der Schweiz. Ein Großteil seiner "Radiopredigten" ist in Buchform veröffentlicht (Tyrolia-Verlag Innsbruck). 
verstorbene Kapuziner Padre Mariano. Und diese Erfahrung, eine treue Gemeinde zu haben, ermutigt den Prediger, wenn ihn die unvermeidliche Sorge quält, ob er nicht ins Leere spreche. Die große und treue Gemeinde gibt dem religiösen Sprecher auch den nötigen Rückhalt gegenüber der Programmleitung, die ja auch daran interessiert ist, den Wünschen der Hörer zu entsprechen.

Seine unsichtbare Gemeinde kann der Rundfunksprecher aus den spontanen Zuschriften kennenlernen, an denen es ihm gewiß nicht fehlen wird. Freilich wird er aus diesen Zuschriften sofort jene beiseite legen, die von jenen Graphomanen geschrieben sind, die nur aus Langeweile einen antwortenden Adressaten suchen. Trotzdem gibt es eine Menge Zuschriften, die dem Rundfunkprediger Aufschlüsse über seine Gemeinde geben. Er wird z. B. sofort aus der Zahl der jeweiligen Zuschriften erfahren, welche Ansprachen besonders angekommen sind. Er erfährt aber auch manches über die religiöse, gesellschaftliche und private Situation seiner Zuhörer.

Eine noch zuverlässigere Quelle des Kennenlernens aber scheint mir die persönliche Begegnung zu sein, die sich auf der Straße, in der Eisenbahn und bei gelegentlichen Vorträgen da und dort ereignet. Die größte Überraschung wird dem Sprecher die Erfahrung sein, wie vielgestaltig tatsächlich seine Gemeinde ist. Der Bogen spannt sich von geistig Anspruchsvollen, von Männern und Frauen hoher Kultur, von Universitätsprofessoren, Managern der Wirtschaft bis zur schlichten Bäuerin auf dem einsamen Berghof. Dabei wird es den Prediger vielleicht besonders beglücken, festzustellen, daß seine Gemeinde auch religiös durchaus nicht uniform ist. Sein Wort überschreitet alle Konfessionsgrenzen, und es wird ihn besonders beglücken zu erfahren, daß sein Wort auch viele erreicht, die am Rand des Glaubens stehen, Zweifler, Verärgerte, ,anonyme Christen', Menschen, die sonst jedem religiösen Anruf unzugänglich sind, in der Stille ihres Zimmers aber doch das Wort Gottes hören.

Diese Erfahrungen wird ein Radiosprecher vor Augen haben müssen, wenn er daran geht, seine sonntägliche Ansprache vorzubereiten. Er darf in keinem Fall "predigen ${ }^{*}$ - Kirchenpredigt ist etwas anderes als die Verkündigung im Radio. Im Französischen hat man die aufschlußreiche Bezeichnung "Causerie religieuse". Es wäre freilich ein Irrtum, dies mit religiöser Plauderei zu übersetzen. Aber das Wort besagt doch, daß der Sprecher mit seinem Zuhörer in einen gelösten Dialog kommt. Er wird versuchen müssen, die Welt und ihre Probleme, die Sehnsüchte des Menschenherzens, letztlich die Frage nach Sinn des Lebens und Sterbens, die Frage nach Gott - aber auch die Antwort des Glaubens, der Offenbarung und Heilsgeschichte mit den Augen seiner mutmaßlichen Zuhörer zu betrachten, sich in ihre Situation hineinzudenken, um ihnen nicht Unverdauliches zuzumuten.

Der Sprecher darf nicht nur plaudern, aber auch nicht nur humanistische Redensarten aufsagen. Er darf nicht in einer Allerweltsethik stecken bleiben, denn er steht ja als Verkündiger der Botschaft Jesu Christi vor dem Mikrophon. Er wird ferner nicht nur Information und das Angebot des christlichen Gedankengutes nur sachlich und nüchtern liefern dürfen. Er muß das Zeugnis des Glaubens geben, das Zeugnis aber will überzeugen. Der Hörer wird die kühle, nüchterne Leidenschaft des Glaubens aus seinen Worten spüren müssen - Gott bewahre ihn vor falschem Pathos, das vor dem Mikrophon schon gar nichts verloren hat. Doch muß beim Sprecher der ganze Ernst spürbar sein, mit dem er das ganze Evangelium - und hier liegt die Betonung auf ,ganze - verkündet. Gerade bei der Pluralität der Zuhörer liegt die Gefahr nahe, ernste Themata zu unterschlagen und sich auf jene Seiten des Evangeliums zu be- 
schränken, über die sich ohnehin die ganze Welt einig ist. Der Sprecher wird den ganzen Ernst der Heilsbotschaft darlegen, auch wenn er immer wieder auf die Fassungskraft seiner Zuhörer Rücksicht nehmen muß.

In keinem Fall wird der Prediger dem Zuhörer unausgekochte Denkmodelle allzu moderner Theologen servieren oder die Gemeinde mit den neuesten theologischen Gags schocken dürfen. Es wäre unverantwortlich, das Mikrophon zu weiterer religiöser Verunsicherung zu mißbrauchen. Die unvermeidliche Pluralität der übrigen Sendungen schafft ohnedies schon Unsicherheit genug. Hier liegt es an der Verkündigung, die Antwort des Glaubens zu geben, aus der der Zuhörer Klarheit und Sicherheit gewinnt. Ich habe den Eindruck, daß manche Kirchenfunkmänner in der Berufung der religiösen Sprecher etwas zu ,großzügig' und ,weitherzig sind. Gerade darauf aber müßte große Sorgfalt verwendet werden.

Ein besonderer Fall ist die religiöse Sendung im Fernsehen. Das Bild in der Nahaufnahme demaskiert unbarmherzig alle eventuellen Schwächen des Sprechers. Der Fernsehprediger wird sich besonders überlegen müssen, wo denn seine Gemeinde zu finden ist: nicht in den Wohnungen der geistigen Elite, sondern am Stadtrand, in den Häusern der kleinen Angestellten, der Beamten, der Arbeiter. Ein Blick aus dem Fenster der Eisenbahn am Stadtrand zeigt ihm sehr klar, wo die Fernsehgeräte stehen, und er wird auch den Ton seiner Botschaft darnach ausrichten müssen. Natürlich wird er sich eines gewissen geistigen Niveaus befleißigen, aber man muß ihn immer auch noch in den Mietskasernen und am Stadtrand verstehen.

Bei der Erarbeitung einer religiösen Sendung wird sich der Sprecher immer wieder fragen müssen, was denn seine Zuhörer gerade jetzt brauchen, worauf sie Antwort haben möchten, was sozusagen heilsgeschichtlich fällig ist. Eine regelmäßige Sendung bringt immer die Gefahr der Eintönigkeit mit sich - umso notwendiger ist es, sich nicht auf einen besonderen Typ biblischer, dogmatischer oder liturgischer Themata festzulegen. Ich halte es besonders für angezeigt, zuweilen auch einmal von einem heroischen Christen, von einem Heiligen zu sprechen.

Der spanische Radioprediger Venancio Marcos hat seinerzeit bei einer Unda-Tagung die exegetische Predigt mit viel Temperament abgelehnt. Er hielt seine eigene Art, in der Sendung mit einer Gruppe von Christen zu sprechen, die er jeweils durch einen Sprecher darstellen ließ, etwa mit einem religiös Liberalen, einer hyperfrommen Seele oder einem Spießbürger, für besonders ansprechend. Er sprach jedenfalls damit in Spanien an, zumal er sich auch nicht scheute, heiße Eisen anzupacken. Für ihn, so meinte er, sei eine Radiopredigt ein Kunstwerk und der Sprecher müsse ein Künstler sein. Sicherlich kann das der Sache dienen - aber noch wichtiger scheint mir zu sein, daß sich der Prediger als Diener am Wort fühlt und aus einem tiefen Glauben spricht.

\section{S U M M A R Y}

For 27 years Father Heinrich Suso Braun has been preaching successfully in the radio especially in Austria and Switzerland. In considering the invisible parish of radio preachers he stresses the importance of knowing his listeners. He gets to know them through letters but mostly through personal contact. Among other things he sais that everything has to be geared to the needs of the listeners and that the preacher should not preach but rather have an entertaining dialogue with his listeners. By this he can proclaim in full the Gospel without necessarily hiding unpleasant subjects. Furthermore preachers should not present undeveloped theological theories of so called modern Theology but should give the listeners clean and concise answers of Christian belief based on sound Faith. 


\title{
RESUME
}

Depuis 27 ans, le Père Heinrich Suso Braun prêche avec succès à la radio, principalement en Autriche et en Suisse. Dans ses réflexions fondamentales à la communauté invisible du prédicateur de radio, il lui importe surtout de connaître l'écouteur. Il fait la connaissance de celui-ci par correspondance, ou mieux encore, en le rencontrant et en s'entretenant avec lui. Tout doit être exécuté en fonction des besoins de l'écouteur avec lequel le prédicateur de radio doit non pas "prêcher", mais mener un dialogue presque divertissant. Mais il a en même temps tout l'Evangile à proclamer et ne doit pas non plus reculer devant les thêmes désagréables. D’autre part, le prédicateur de radio ne doit pas non plus choquer sa communauté avec "d'hardis modèles de pensée de théologiens modernes" ou des gags de théologiens. Sa tâche consiste plutôt à donner à l'écouteur la réponse de la foi à partir de son propre coeur de croyant, réponse par laquelle il gagne en clarté et en sûreté.

\section{R E S U M E N}

Desde hace 27 años predica con éxito a través de las ondas, sobre todo en Austria y Suiza, el Padre Heinrich Suso Braun. Para él lo más importante al considerar la relación fundamental del predicador radiofónico con su comunidad invisible es el conocimiento que se tiene del radioescucha. Este conocimiento se adquiere a través de la correspondencia y mejor aún mediante el trato personal y la conversación. Se debe enfocar todo teniendo en cuenta las necesidades del oyente, ante el que el predicador radiofónico no debe „predicar“. Antes bien debe mantener con él un diálogo casi entretenido. Por otra parte debe anunciar el Evangelio completo y no puede dejarse intimidar por temas incómodos. El predicador radiofónico debe abstenerse además de impresionar a su comunidad con modelos de pensamiento "poco maduros" de teólogos modernos ó con trucos teológicos. Por el contrario debe proporcionar al creyente la respuesta de la fe, que le ayude a ganar claridad y seguridad.

\section{„Unser Führer ist der Heiland”: Meinungsforschung im Dritten Reich ${ }^{1}$}

\author{
von Ulrich Kröll
}

$\mathrm{Zu}$ den konstituierenden Elementen des modernen demokratischen Staates gehören die Meinungsfreiheit (eingeschlossen die Freiheit, Meinungen durch publizistische Medien zu multiplizieren) sowie die politische Willensbildung durch die Regierten. Die Diskussion politisch und gesellschaftspolitisch relevanter Sachverhalte in direkter oder vermittelter Kommunikation durch Individuen und soziale Gruppen - als Vorstufe oder begleitend zur demokratischen Willensbildung - weckt bei politischen, gesellschaftlichen und wirtschaftlichen Interessengruppen den Wunsch, vorherrschende und abweichende Meinungen, d.h. die ebenso vielzitierte wie umstrittene, öffentliche Meinung', zu erforschen oder gar zahlenmäßig abgesichert empirisch zu erheben, um so den ,Markt' für politische Maßnahmen, für Gruppeninteressen, für wirtschaftliche Produkte abzuklären. Meinungsforschung in einem demokratisch-rechtsstaatlichen System geht dabei oft von der unausgesprochenen Prämisse aus, daß Informationen und kontroverse Meinungen zu einem Punkt dem Interessierten zugänglich sind und ihn auch erreichen und $\mathrm{da}$ der Interessierte (d. h. der mündige, politisch bewußte

Dr. Ulrich Kröll, wiss. Mitarbeiter am Deutschen Institut für wissenschaftliche Pädagogik, hat seine Studien mit einer Dissertation über "Die internationale Buren-Agitation 1899-1902“ (Münster 1973) abgeschlossen. 